\title{
An Assessment of Dosimetric Characteristics of Inline 2.5 Mega Voltage Unflattened Imaging X-Ray Beam
}

\author{
Tamilarasan Rajamanickam ${ }^{1,2}$, Sivakumar Muthu', Perumal Murugan", \\ Chinnaiah Dinesan ${ }^{1}$, Chandrasekaran Mekala ${ }^{3}$, Krishnamoorthy Senthilnathan ${ }^{2}$, \\ Narayanasamy Arunai Nambi Raj ${ }^{4}$, Padmanabhan Ramesh Babu ${ }^{2 *}$
}

\begin{abstract}
Purpose: The aim of this work is to study the dosimetric parameters of newly introduced $2.5 \mathrm{MV}$ imaging x-ray beam used as inline imaging to do setup verification of the patient undergoing radiation therapy. As this x-ray beam is in megavoltage range but comprises of a lower energy spectrum. It is essential to study the pros and cons of 2.5 MV imaging X-ray beam for clinical use.Methods: The mean energy was calculated using the NIST XCOM table through MAC. Profile analysis was done using RFA to understand the percentage depth dose, degree of unflatteness, symmetry, penumbra and out of field dose. Dose to skin for the $2.5 \mathrm{MV}$ x-ray beam was analysed for field sizes $10 x 10$ $\mathrm{cm}^{2}, 20 \times 20 \mathrm{~cm}^{2}, 30 \times 30 \mathrm{~cm}^{2}$. Leakage measurements for treatment head and at the patient plane were done using IEC $819 / 98$ protocol. Finally, the spatial resolution and contrast were analyzed with and without patient scatter medium. Results: The MAC at $15 \mathrm{~cm}$ off-axis was found to be lower than that at the CAX. Similarly, there was a decrease in mean energy from $0.47 \mathrm{MV}$ to $0.37 \mathrm{MV}$ at $15 \mathrm{~cm}$ off-axis. The reduction of mean energy towards off-axis is lower than the other high energy MV x-ray beams. The tuned absolute dose of $1 \mathrm{cGy} / \mathrm{MU}$ is consistent and within $< \pm 1 \%$. The relative output factors were found to be in correlation with Co-60. The beam quality of $2.5 \mathrm{MV}$ x-ray beam was found to be 0.4771 . The profile parameters like the degree of unflatness of the $2.5 \mathrm{x}$-ray beam were studied at $85 \%, 90 \%$, $95 \%$ lateral distances, and the penumbra at different depth and field sizes are higher than the $6 \mathrm{MV}$ treatment beam. In addition, out of field dose also drastically increases to a maximum of up to $30 \%$ laterally at $5 \mathrm{~cm}$ at deeper depths. The skin dose increases from $48.51 \%$ to $88.15 \%$ from $6 \mathrm{MV}$ to $2.5 \mathrm{MV}$ x-ray beam for the field size $10 \times 10 \mathrm{~cm}^{2}$. Also, the skin dose increases from $88.15 \%$ to $91.78 \%$ from the field size $10 \times 10 \mathrm{~cm}^{2}$ to $30 \times 30 \mathrm{~cm}^{2}$. Although the measured leakage radiation for $2.5 \mathrm{MV}$ x-ray beam at the patient plane and other than patient planes are with the tolerance limit, an increase in exposure towards gantry side compared to other areas around treatment head and the patient plane may lead to more skin dose to head and chest while imaging pelvis region. The MLC transmission of 2.5 MV x-ray beam such as inter, intra and edge effect are $0.40 \%, 0.37 \%$ and $11 \%$ respectively. The spatial resolution of $2.0,1.25$ and $0.9 \mathrm{LP} / \mathrm{mm}$ was observed for KV, 2.5MV, and $6 \mathrm{MV}$ x-ray beams. The spatial resolution and contrast of $2.5 \mathrm{MV}$ x-ray beam are superior to $6 \mathrm{MV} \mathrm{x}$-ray beam and inferior to KV x-rays. Conclusions: The $2.5 \mathrm{MV}$ x-ray imaging beam is analysed in view of beam characteristics and radiation safety to understand the above-studied concepts while using this imaging beam in a clinical situation. In future, if $2.5 \mathrm{MV}$-ray beam is used for treatment purpose with increased dose rate, the above-studied notions can be incorporated prior to implementation.
\end{abstract}

Keywords: $2.5 \mathrm{MV}$ x-ray beam- mean energy- skin dose- leakage radiation- spatial resolution- contrast

Asian Pac J Cancer Prev, 20 (8), 2531-2539

\section{Introduction}

The main objective of radiotherapy treatment is to give maximum dose to the tumor and minimal dose to nearby critical organs. Implications of missing tumor lead to lowering tumor control probability tend to lower the survival rate. The probability of normal tissue complication also increases considerably depending on the degree of dose misadministration (Bentzen et al., 2010). Thus, the patient setup verification is a vital part of delivering radiation therapy. In current clinical practice, the pre-treatment imaging is a significant one to verify the patient setup and organ movement. Historically, films mounted in cassettes were kept behind the patient in the beam direction and orthogonal films were taken to verify the setup accuracy (Marks et al., 1976) which proved

${ }^{1}$ Department of Radiation Oncology, Sri Shankara Cancer Hospital and Research Centre, Bengaluru, Karnataka, ${ }^{2}$ Department of Physics, SAS, ${ }^{4}$ Centre for Biomaterials, Cellular and Molecular Theranostics, Vellore Institute of Technology, Vellore, Tamil Nadu, India, ${ }^{3}$ Principal Clinical Scientist and Stereotactic Lead, University Hospital Southampton NHS FT, Tremona Rd, Southampton SO166YD, UK. *For Correspondence: prameshbabu@vit.ac.in 
to be a time-consuming technique. With the advent of new technology with electronic portal imaging devices (EPID) such as liquid ionization and amorphous siliconbased detectors operated remotely has enhanced the treatment accuracy with considerably reduced imaging time (Michael et al., 2001; Bel, et al., 1996; Pisani et al., 2000; Antonuk et al., 2002). The megavoltage energy used for treatment (i.e. $6 \mathrm{MV}$ x-ray) were utilized to take setup verification (Quanshi et al., 2016). At these energies, the Compton scattering cross section becomes a dominant interaction process at $20 \mathrm{KeV}$ for soft tissues and above 50 $\mathrm{KeV}$ for bones $(\sim \mathrm{Zeff}=13)$. It depends on electron density of a material except for hydrogen, which varies only slightly with atomic number. Hence there is a reduction in contrast significantly at megavoltage energies. Whereas at low energies, photoelectric cross section is predominant which is proportional to $\mathrm{Z}^{3}$ and inversely proportional to energy $\left(1 / \mathrm{E}^{3}\right)$ (Khan, 1994).

To overcome the problem of this megavoltage Compton cross section, all vendors came up with kilovoltage imaging system isocentrically placed plane perpendicular to the megavoltage beam direction by means of the predominant photoelectric cross section at kilovoltage energies can be utilized hence improving contrast and spatial resolution of orthogonal imaging and better patient setup (Yin et al., 2009; Verellen et al., 2008). Recently, as a part of the imaging technique, a $2.5 \mathrm{MV}$ inline imaging beam was introduced in the clinical setup for better imaging compared to $6 \mathrm{MV}$ x-ray beam. Owing to the reduction of $\mathrm{x}$-ray beam energy (soft spectrum) moves the most predominant interaction zone from Compton to photoelectric cross-section; better imaging contrast is obtained (James et al., 2016).

A novel low energy $2.5 \mathrm{MV}$ x-ray imaging beam is made available as in-line portal image verification of the patient setup in TrueBeam linear accelerator. The $2.5 \mathrm{MV}$ x-ray beam is produced by a $2.5 \mathrm{MeV}$ monoenergetic electron beam focused on a $2 \mathrm{~mm} \mathrm{Cu}$ target (Grace et al., 2016). The coincidence of isocentre of megavoltage and kilovoltage beam is important for precise image-guided radiotherapy (IGRT). Therefore, high accuracy and effective quality assurance is required to maintain megavoltage and kilovoltage isocenter within tolerance (Herman et al., 1994; Dawson et al., 2006). However, 2.5 MV x-ray imaging beam is in-line with the treatment beam so it is perfectly aligned with treatment isocenter. Unlike other traditional megavoltage beams, 2.5 MV x-ray beam operates without flattening filter with single nominal dose rate $(60 \mathrm{MU} / \mathrm{min})$ and this dedicated $2.5 \mathrm{MV}$ x-ray beam is controlled by the monitor chambers of the Clinac. Thus, it must be calibrated in the same way as treatment beams. The customization of this $2.5 \mathrm{MV}$ x-ray beam for treatment purpose is not yet practiced worldwide since there is not enough study done in understanding the production of low energy beams with low $\mathrm{Z}$ targets with required treatment dose rate $(\sim 400 \mathrm{MU} / \mathrm{min})$.

This novel 2.5 MV x-ray imaging beam need to be commissioned similar to other megavoltage treatment beams and can be used clinically only after proper approval from the competent authority. In our study, we analyse critical parameters such as mean energy through mass attenuation coefficient at central and off-axis, degree of unflatness and symmetry, penumbra and out of field dose through beam profile, percentage depth dose (PDD), output factor, skin dose, leakage radiation in the patient plane and other than patient plane, MLC transmission, contrast and spatial resolution.

\section{Materials and Methods}

The experiment was carried out in TrueBeam 2.0 (Varian Medical System Inc., Palo Alto, CA, USA) capable of delivering flattened and unflattened x-ray beams of 6 $\mathrm{MV}, 10 \mathrm{MV}, 15 \mathrm{MV}$, and $2.5 \mathrm{MV}-\mathrm{FFF}, 6 \mathrm{MV}-\mathrm{FFF}, 10$ MV-FFF respectively. The recently commissioned $2.5 \mathrm{MV}$ FFF beam with a dose rate of $60 \mathrm{MU} / \mathrm{min}$ is used as an MV imaging (along with $6 \mathrm{MV}$ imaging) and remaining other energies are utilized for treatment purposes. It is equipped with an amorphous silicon array detector (aSi-1000) used for image verification.

The penetration ability of the beam i.e., mass attenuation coefficient $\mu / \rho\left(\mathrm{cm}^{2} / \mathrm{g}\right)$ of $2.5 \mathrm{MV}$ x-ray beam (Kerur et al., 1993; Jackson et al., 1981) using titanium Grade 5 (Eng, 2000; Weisneret al., 2013) high-Z material (Table 1) $\left(\rho=8.6 \mathrm{~g} / \mathrm{cm}^{2}\right)$ was determined by narrow beam geometry. The measurement was carried out in air using CC13 ionization chamber for 500 MU's with an appropriate build-up (5mm) to avoid electronic disequilibrium. The chamber was positioned at isocenter and titanium high- $Z$ material placed $10 \mathrm{~cm}$ above the chamber level exactly, equally shadowing around the chamber. A field size of $3 \times 3 \mathrm{~cm}^{2}$ was opened and ensured the field border was inside the high- $Z$ material. The gap in between chamber and high- $Z$ material was kept in such a way that it was good enough to avoid any scattering electrons to evade overestimation of the result. The measurement was carried out both in the central axis and off-axis of about $15 \mathrm{~cm}$ from CAX in an inline direction to determine variation in mean energy at off-axis. The measured mass attenuation coefficients were compared with NIST mass attenuation cross-section data given for particular high-Z material (Berger et al., 1987; Gerward et al., 2004). The mean energy was interpolated from given NIST mass attenuation cross-section data for particular high-Z material and compared to the Monte Carlo derived mean energy derived by Ding et al., (2018).

Although the $2.5 \mathrm{MV}$ x-ray beam is in megavoltage range and used only for imaging purposes, the beam needs to be tuned to deliver constant dose for each monitor unit (MU) as it is controlled by monitor chamber. Hence, an absolute dose calibration was carried out using farmer ion chamber (FC65G) and Dose1 electrometer (IBA Dosimetry, Germany). As per TRS 398, the beam quality measured for $2.5 \mathrm{MV}$ is slightly lower than $\mathrm{CO}^{60}$ beam quality. Hence, for calibration purpose, we chose the beam quality conversion factor $\left(\mathrm{K}^{\mathrm{Q}}\right)$ as unity $\left(\mathrm{K}^{\mathrm{Q}}=\right.$ 1.000). The output of $2.5 \mathrm{MV}$ beam was calibrated as $1 \mathrm{cGy} / \mathrm{MU}$ at dmax for the field size of $10 \times 10 \mathrm{~cm}^{2}$ for $100 \mathrm{~cm}$ SSD in water. The absolute dose measurement of 2.5 MV imaging beam is done on a monthly basis to check the output stability of the x-ray beam (TRS No. $398,2000)$. The output factor (Sc,p) of the x-ray beam 
depends on the target, monitor chamber, flattening filter, the field defining jaws, MLC as well as the energy of that particular x-ray beam. Since the studied energy is very low compared to $6 \mathrm{MV}$ treatment beam used for imaging purpose, the scattering properties will vary drastically for 2.5 MV than 6 MV x-ray beam (Sauer et al., 2007; Abdullah et al., 2018). The output factor (Sc,p) for $2.5 \mathrm{MV}$ imaging beam was measured with the help of CC13 ion chamber placed at $5 \mathrm{~cm}$ depth in the PMMA phantom at $95 \mathrm{~cm}$ SSD. Measurements were done for the field sizes $3 \times 3 \mathrm{~cm}^{2}$ to $40 \times 40 \mathrm{~cm}^{2}$ and analysed against Co-60, $6 \mathrm{MV}$ $\mathrm{FF}$ and $6 \mathrm{MV}$ UF $\mathrm{x}$-ray beams. The penetration power or otherwise beam quality of $2.5 \mathrm{MV}$ imaging beam is defined as per TPR ${ }^{20,10}$ (Sharma et al., 2008). Absolute dose measurements were done with chamber placed isocentrically at $100 \mathrm{~cm}$ and depth of 10 and $20 \mathrm{~cm}$ from the water surface. Beam profiles were measured using a RFA (Blue Phantom2 RFA - IBA Dosimetry, Germany) and $\mathrm{CC} 13$ ionization chambers. The beam penumbra (distance between $80 \%-20 \%$ ), degree of unflatness and symmetry were analysed with the help of profiles taken for different field sizes and depths (Sahani et al., 2013).

The skin dose for 2.5 MV x-ray beam was analysed to understand the skin sparing effect (Devic et al., 2006; Sharma et al., 2013). Although we use very few MUs for taking images it is important to analyse the add-on skin dose along with treatment dose. The skin dose measurement was carried out with the help of a parallel plate chamber (PPC40) in PMMA phantom at every $1 \mathrm{~mm}$ from the surface $(0 \mathrm{~cm})$ to $10 \mathrm{~mm}$ for the filed sizes $10 \times 10 \mathrm{~cm}^{2}, 20 \times 20 \mathrm{~cm}^{2}, 30 \times 30 \mathrm{~cm}^{2}$ as field opening may vary during the imaging procedure. The amount of radiation exposed other than treatment area while imaging using 2.5 MV x-ray beam was studied as the low energy photons absorbed more at skin than $6 \mathrm{MV}$ megavoltage X-ray beam used for imaging (Purdy et al., 1993; Sahani et al., 2009). Leakage measurements such as head leakage and leakage in the patient plane were measured using IEC 618/92 protocol for 2.5 MV x-ray beam using fluke survey meter at 32 points for 10 MUs as shown in Figure 5. The MLC leakage test was done using CC13 chamber and PMMA phantom for 500 MUs at $0.5 \mathrm{~cm}$ depth selected because of low energy x-rays and deeper depth will make an improper signal in chamber leads to imperfect reading (Mark et al., 2000). The contrast and spatial resolution for 2.5 MV x-ray beam are measured with the help of Las Vegas phantom and Leeds phantom and compared with other megavoltage (6 MV-FF) and kilovoltage (80 KV, $2.5 \mathrm{mAs}$ ) energies with and without overlaying scatter which imitate the patient condition (Nithya et al., 2015).

\section{Results}

Mass Attenuation Coefficient and Mean Energy

The mass attenuation coefficient $(\mu / \rho)$ measured for 2.5 MV imaging $\mathrm{x}$-ray beam at central axis and off-axis of about $15 \mathrm{~cm}$ is $0.08453 \mathrm{~cm}^{2} / \mathrm{g}$ and 0.10214 $\mathrm{cm}^{2} / \mathrm{g}$ respectively. The attenuation cross section was calculated using XCOM software version 3.1 for an exact combination of the high $\mathrm{Z}$ material for monoenergetic
Table 1. Physical and Chemical Properties of Titanium Grade 5 Alloy

\begin{tabular}{cccccc}
\hline Material & $\begin{array}{l}\text { Chemical } \\
\text { Composition }\end{array}$ & $\begin{array}{c}\% \text { of } \\
\text { Composition }\end{array}$ & Z & A & $\begin{array}{c}\rho \\
\left(\mathrm{g} / \mathrm{cm}^{3}\right)\end{array}$ \\
\hline Titanium (Grade 5) & & & & \\
Titanium (Ti) & $89.55 \%$ & 22 & 48 & 4.5 \\
Aluminum (Al) & $6.00 \%$ & 13 & 27 & 2.7 \\
Vanadium (V) & $4.00 \%$ & 23 & 50.9 & 6.1 \\
Iron (Fe) & $0.25 \%$ & 26 & 56 & 7.9 \\
Oxygen (O) & $0.20 \%$ & 8 & 16 & 1.43 \\
\hline
\end{tabular}

Table 2. Absolute Dose Calibration Parameter of 2.5 MV X-Ray Beam

\begin{tabular}{lc}
\hline Parameter & $2.5 \mathrm{MV}-\mathrm{UF}$ \\
\hline$K_{s}$ & 0.9637 \\
$K_{p o l}$ & 1.0501 \\
$P D D(10 \mathrm{~cm})$ & 54.2 \\
$D_{\max }$ & $0.5 \mathrm{~cm}$ \\
$K_{O}$ & 1.000 \\
\hline
\end{tabular}

$\mathrm{x}$-ray beams from $1 \mathrm{KeV}$ to $100 \mathrm{GeV}$. An equivalent mean energy calculated from the attenuation cross section table for central axis and $15 \mathrm{~cm}$ off-axis is $0.47 \mathrm{MeV}$ and 0.32 $\mathrm{MeV}$ respectively. The Monte Carlo simulated mean energy at the central axis calculated by Ding et al., (2018) is $0.48 \mathrm{MeV}$. The central axis mean energy by Monte Carlo and attenuation cross-section methods are in agreement with each other. The low energy component in the beam spectrum makes the mean energy in kilovoltage range and makes the imaging with better contrast and spatial resolution.

\section{Beam Quality and Absolute Dose Calibration}

The beam quality measured in terms of tissue phantom ratio $\left(\mathrm{TPR}^{20,10}\right)$ by farmer ion chamber for $2.5 \mathrm{MV}$ imaging $\mathrm{X}$-ray beam is 0.4771 . The absolute dose calibrated for 2.5 MV-UF imaging beam is done using the following parameter (Table 2)

The beam is tuned to deliver $1 \mathrm{cGy} / \mathrm{MU}$ at the dose

Table 3. Measured Build-up Dose for Different Field Sizes for 2.5 MV X-Ray Beam

\begin{tabular}{lccc}
\hline Depth $(\mathrm{mm})$ & \multicolumn{3}{c}{$\%$ of Dose at Build-up Region } \\
& $10 \times 10 \mathrm{~cm}^{2}$ & $20 \times 20 \mathrm{~cm}^{2}$ & $30 \times 30 \mathrm{~cm}^{2}$ \\
\hline 0 & $88.15 \%$ & $89.98 \%$ & $91.78 \%$ \\
1 & $95.35 \%$ & $95.86 \%$ & $96.50 \%$ \\
2 & $97.78 \%$ & $97.75 \%$ & $97.97 \%$ \\
3 & $98.73 \%$ & $98.37 \%$ & $98.39 \%$ \\
4 & $99.09 \%$ & $98.72 \%$ & $98.70 \%$ \\
5 & $100.00 \%$ & $100.00 \%$ & $100.00 \%$ \\
6 & $99.84 \%$ & $99.83 \%$ & $99.89 \%$ \\
7 & $99.72 \%$ & $99.73 \%$ & $99.81 \%$ \\
8 & $99.43 \%$ & $99.61 \%$ & $99.71 \%$ \\
9 & $99.21 \%$ & $99.48 \%$ & $99.64 \%$ \\
10 & $98.95 \%$ & $99.36 \%$ & $99.48 \%$ \\
\hline
\end{tabular}

Asian Pacific Journal of Cancer Prevention, Vol $20 \mathbf{2 5 3 3}$ 


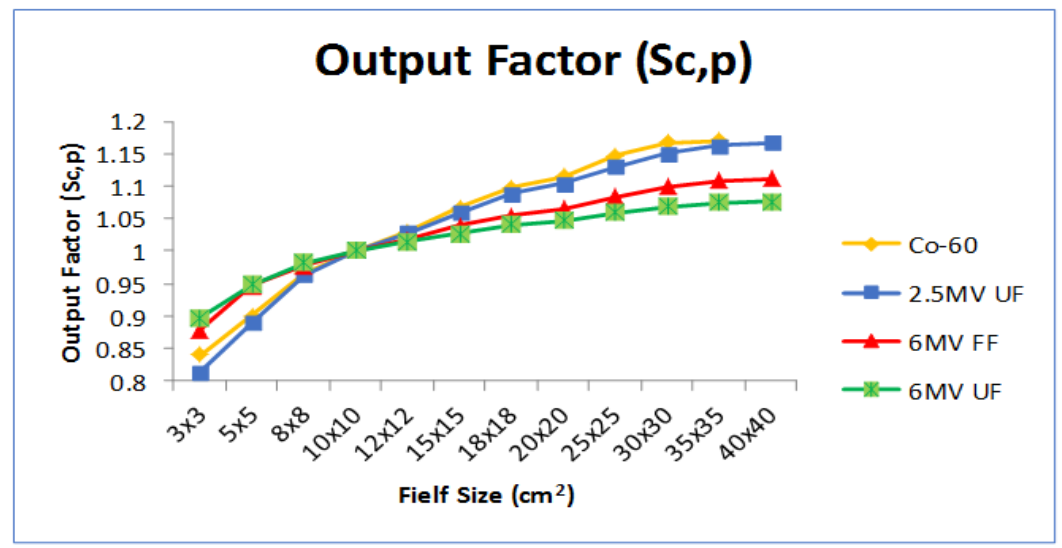

Figure 1. Measured Output Factor (Sc,p) for different Energies (MV) and Field Sizes $\left(\mathrm{cm}^{2}\right)$

Table 4. Measured Head Leakage for 2.5 MV X-Ray Beam

\begin{tabular}{lcccc}
\hline \multicolumn{5}{c}{ Head Leakage Measurement } \\
Points & Air Kerma Level at $5 \mathrm{~cm}$ & Air Kerma Level at $1 \mathrm{~m}$ \\
\hline A & 1.8 & 0.02 & 1 & 0.01 \\
B & 1.6 & 0.02 & 1 & 0.01 \\
C & 2.5 & 0.03 & 1.2 & 0.01 \\
D & 2 & 0.02 & 1.2 & 0.01 \\
E & 8 & 0.08 & 3.5 & 0.04 \\
F & 19 & 0.19 & 4.4 & 0.04 \\
G & 1.1 & 0.01 & 1.2 & 0.01 \\
H & 1.9 & 0.02 & 2 & 0.02 \\
I & 2.1 & 0.02 & 2.1 & 0.02 \\
J & 1 & 0.01 & 1.2 & 0.01 \\
K & 22 & 0.22 & 5.4 & 0.05 \\
L & 8.6 & 0.09 & 3.2 & 0.03 \\
Avg Leakage & 0.06 & & 0.02 \\
Max Leakage & 0.22 & & 0.05 \\
\hline
\end{tabular}

rate of $60 \mathrm{MU} / \mathrm{min}$. An absolute dose output consistency of $< \pm 1 \%$ is observed every month.

\section{Output Factor (Sc,p)}

The measured output factor for 2.5 MV x-ray beam for various collimator settings (field sizes) in Figure 1 shows that the output factor increases with an increase in field size. Relative output factors for 2.5 MV energy is lower than other treatment energies such as Co-60, 6MV flattened and unflattened x-ray beams below reference field size $\left(10 \times 10 \mathrm{~cm}^{2}\right)$. The output factor increases for field sizes greater than reference field size.

An increase in output factor for higher filed sizes is due to the contribution of lateral scattering of low energy photons compared to high energy x-ray beams. Although the flattened $\mathrm{x}$-ray beam has uniform fluence across the field size, the beam hardening in the flattening filter reduces the extent of low energy photons which results in a reduction of lateral scatter contribution. However, for unflattened x-ray beam, the fluence stands non-uniform and the low energy photons are making the contribution to the measurement point. Subsequently, the fluence of the unflattened $\mathrm{x}$-ray beam decreases gradually towards field border, the amount of lateral scatter decreases which makes the output factor lower than the flattened one. Figure 1 show that the output factor for $2.5 \mathrm{MV}$ imaging x-ray beam follows very closely with Co- 60 . The $6 \mathrm{MV}$ unflattened $\mathrm{x}$-ray beams are more forward peaked in central axis and gradually decreases towards field edge whereas the production of 2.5 MV unflattened x-ray beam in the target is mere forward peaked in the central axis and scatters laterally more.

\section{Percentage Depth Dose}

The percentage depth dose measured for $2.5 \mathrm{MV}$

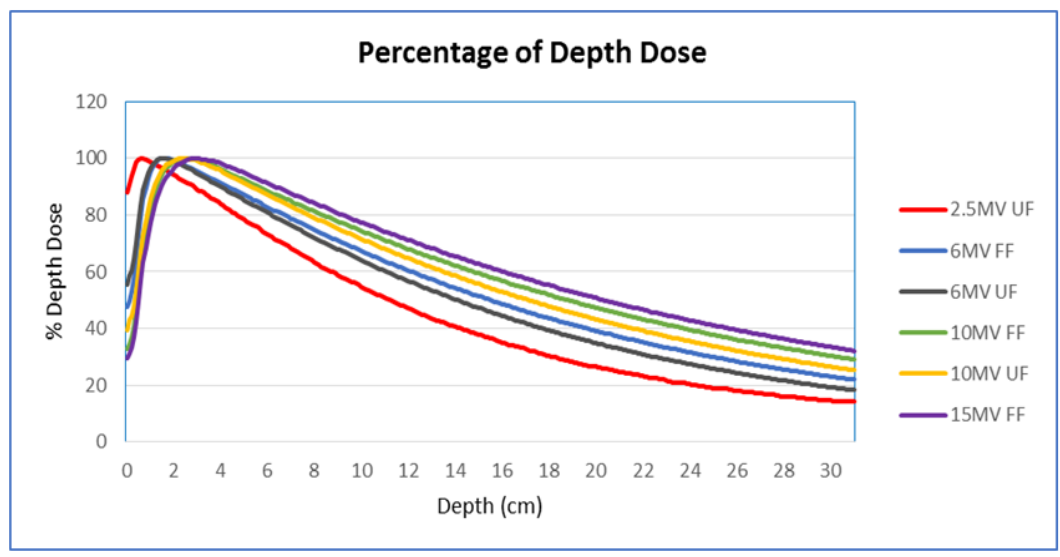

Figure 2. Measured Percentage Depth Dose of 2.5 MV and Compared with Different Energies 
Table 5. Measured Leakage at the Patient Plane for 2.5 \& 6 MV X-Ray Beam

$$
\text { Leakage at Patient Plane (IEC 618/92) }
$$

\begin{tabular}{|c|c|c|c|c|c|}
\hline \multirow[t]{3}{*}{ Distance (m) } & \multirow[t]{3}{*}{ Location } & \multicolumn{4}{|c|}{ Measured Air Kerma (mR) } \\
\hline & & \multicolumn{2}{|c|}{$2.5 \mathrm{MV}$} & \multicolumn{2}{|c|}{$6 \mathrm{MV}$} \\
\hline & & $\mathrm{mR}$ & $\%$ of Leakage & $\mathrm{mR}$ & $\%$ of Leakage \\
\hline \multirow[t]{10}{*}{$(\mathrm{R} 0+0.25(2-\mathrm{R} 0))=0.71 \mathrm{~m}$} & $\mathrm{~A}$ & 0.115 & 0.001 & 1.3 & 0.013 \\
\hline & $\mathrm{B}$ & 0.215 & 0.002 & 0.8 & 0.008 \\
\hline & $\mathrm{C}$ & 0.58 & 0.006 & 1.6 & 0.016 \\
\hline & $\mathrm{D}$ & 0.71 & 0.007 & 1.4 & 0.014 \\
\hline & $\mathrm{E}$ & 1.28 & 0.013 & 1.8 & 0.018 \\
\hline & $\mathrm{F}$ & 0.66 & 0.007 & 1.3 & 0.013 \\
\hline & G & 0.54 & 0.005 & 0.9 & 0.009 \\
\hline & $\mathrm{H}$ & 0.14 & 0.001 & 0.8 & 0.008 \\
\hline & Avg Leakage \% & & 0.005 & & 0.012 \\
\hline & Max Leakage \% & & 0.013 & & 0.018 \\
\hline \multirow[t]{10}{*}{$(\mathrm{R} 0+0.5(2-\mathrm{R} 0))=1.14 \mathrm{~m}$} & I & 0.2 & 0.002 & 0.7 & 0.007 \\
\hline & $\mathrm{J}$ & 0.16 & 0.002 & 0.6 & 0.006 \\
\hline & $\mathrm{K}$ & 0.29 & 0.003 & 0.9 & 0.009 \\
\hline & $\mathrm{L}$ & 0.47 & 0.005 & 0.7 & 0.007 \\
\hline & M & 0.9 & 0.009 & 0.8 & 0.008 \\
\hline & $\mathrm{N}$ & 2.5 & 0.025 & 0.6 & 0.006 \\
\hline & $\mathrm{O}$ & 0.8 & 0.008 & 0.6 & 0 \\
\hline & $\mathrm{P}$ & 0.21 & 0.002 & 0.4 & 0.004 \\
\hline & Avg Leakage \% & & 0.007 & & 0.007 \\
\hline & Max Leakage \% & & 0.025 & & 0.009 \\
\hline \multirow[t]{10}{*}{$(\mathrm{R} 0+0.75(2-\mathrm{R} 0))=1.57 \mathrm{~m}$} & Q & 0.06 & 0.001 & 0.3 & 0.001 \\
\hline & $\mathrm{R}$ & 0.11 & 0.001 & 0.3 & 0.003 \\
\hline & $\mathrm{S}$ & 0.4 & 0.004 & 0.8 & 0.008 \\
\hline & $\mathrm{T}$ & 0.36 & 0.004 & 1 & 0.02 \\
\hline & $\mathrm{U}$ & 2.6 & 0.026 & 1.4 & 0.014 \\
\hline & $\mathrm{V}$ & 0.35 & 0.004 & 1.2 & 0.012 \\
\hline & $\mathrm{W}$ & 0.38 & 0.004 & 0.7 & 0.007 \\
\hline & $\mathrm{X}$ & 0.1 & 0.001 & 0.3 & 0.003 \\
\hline & Avg Leakage \% & & 0.005 & & 0.009 \\
\hline & Max Leakage \% & & 0.026 & & 0.014 \\
\hline
\end{tabular}

megavoltage $x$-ray imaging beam (Figure 2) shows that the maximum dose build-up occurs at $0.5 \mathrm{~cm}$ from the surface which is almost equal to the Co-60 beam. The depth dose value at $10 \mathrm{~cm}$ and $20 \mathrm{~cm}$ are $54.7 \%( \pm 0.1 \%)$ and 26.5
$\%( \pm 0.1 \%)$ respectively.

\section{Beam Profile}

Beam profiles were analysed for 2.5 MV imaging x-ray
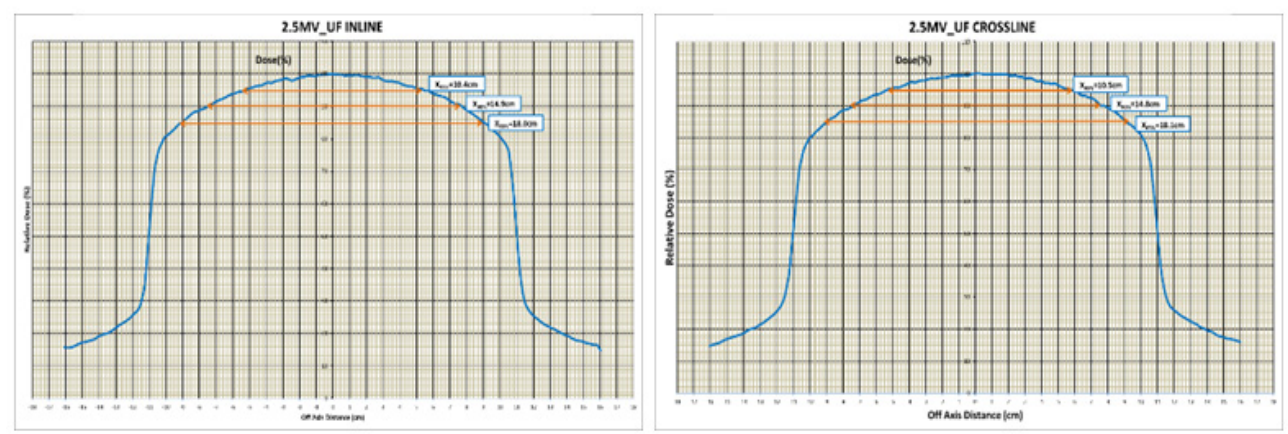

Figure 3. Measured Degree of Unflatness of 2.5 MV X-Ray Beam for Inline and Cross Line Profile 

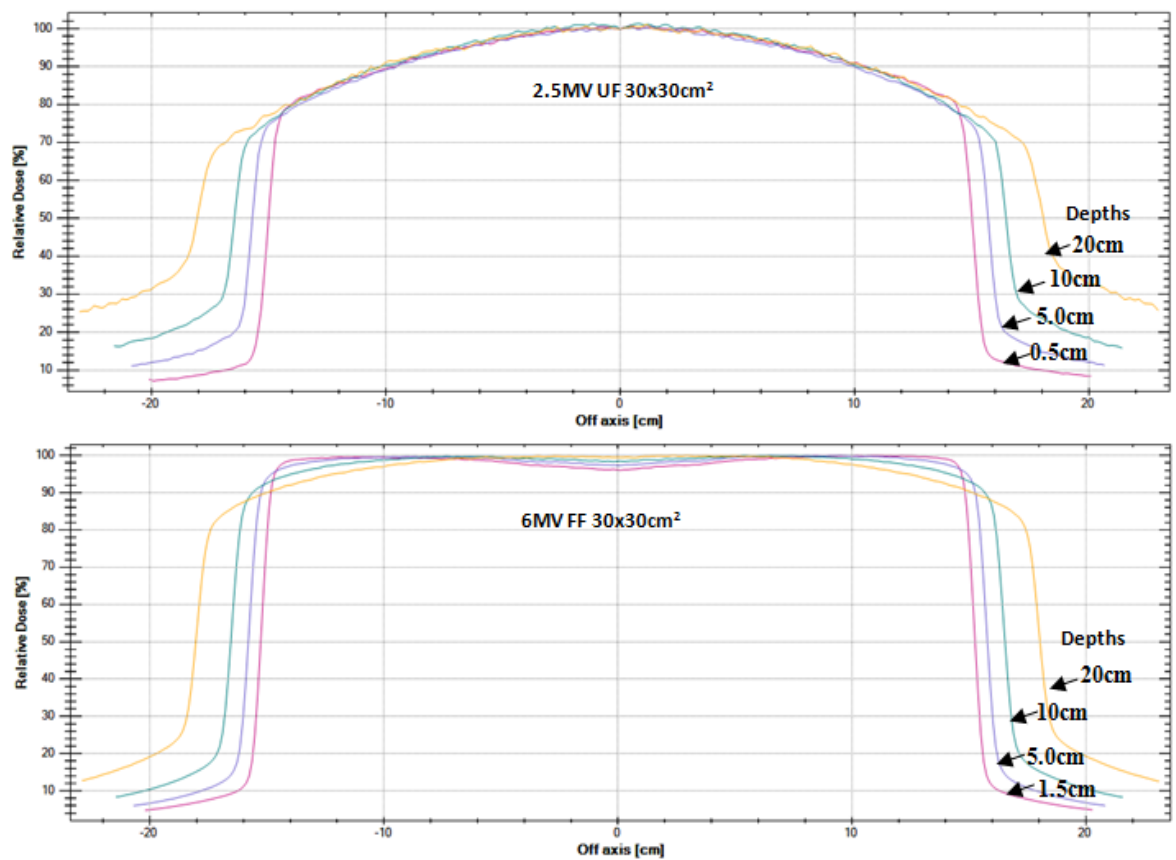

Figure 4. Penumbra and Out of Field Dose Measured Through Profile for Various Depths for 6 and 2.5 MV X-Ray Beams

beam to study parameters such as the degree of unflatness, symmetry, penumbra and out of field dose.

\section{Flatness and Symmetry}

The degree of unflatness of the unflattened x-ray beams is analysed as per Atomic Energy Regulatory Board task group recommendation (Sahani et al., 2014) as shown in Figure 3. It says that the degree of unflatness is the lateral distance from the central axis at $90 \%, 75 \%$ and $60 \%$ dose point on either side of the beam profile. This quantification of the degree of unflatness can be applied only to clinically available energies such as 6 MV UF and 10 MV UF x-ray beams because of the more pronounced peak in the central axis. As the energy increases the forward peak increases in central axis so that the degree of unflatness at $60 \%$ can be obtained with ease. However, in the case of $2.5 \mathrm{MV}$ UF X-ray beams, the degree of unflatness starts from $80 \%$ only. This is due to the fact that when low energy electrons hit the target the Bremsstrahlung angular distribution of x-rays are less forward pronounced (for $100 \mathrm{KeV}$ isotropic). The beam profile for 2.5 MV UF x-ray beams are less peaked and gradually decreases laterally till 80 $\%$ of the central axis and then the beam fall-off occurs. Therefore, we adopted $85 \%, 90 \%$ and $95 \%$ lateral distances from the central axis for analysing the degree of unflatness of 2.5 MV x-ray beam. The same measurement was repeated every month to check the consistency of the degree of unflatness.

The lateral distances measured to find the degree of unflatness for $85 \%, 90 \%$, and $95 \%$ are $18.1 \mathrm{~cm}, 14.8 \mathrm{~cm}$, and $10.6 \mathrm{~cm}$ respectively. The consistency of the unflatness of 2.5 MV imaging $\mathrm{x}$-ray beam shows within $\pm 1 \mathrm{~mm}$.

The symmetry of the $2.5 \mathrm{MV} x$-rays imaging inline and crossline beam are $0.5 \%$ well within the tolerance of $\pm 2 \%$.

\section{Penumbra and Out of field Dose}

Figure 4 shows that the penumbra measured for 2.5 MV x-ray imaging beam at depth of $0.5 \mathrm{~cm}$ (Dmax), 5 $\mathrm{cm}$ and $10 \mathrm{~cm}$ for the range of field sizes from $5 \times 5 \mathrm{~cm}^{2}$ to $30 \times 30 \mathrm{~cm}^{2}$ is $0.7 \mathrm{~cm}$ to $1.42 \mathrm{~cm}, 0.86 \mathrm{~cm}$ to $2.7 \mathrm{~cm}$ and $1.4 \mathrm{~cm}$ to $5.4 \mathrm{~cm}$ respectively. However, penumbra measured for depths beyond $10 \mathrm{~cm}$, the off-axis dose
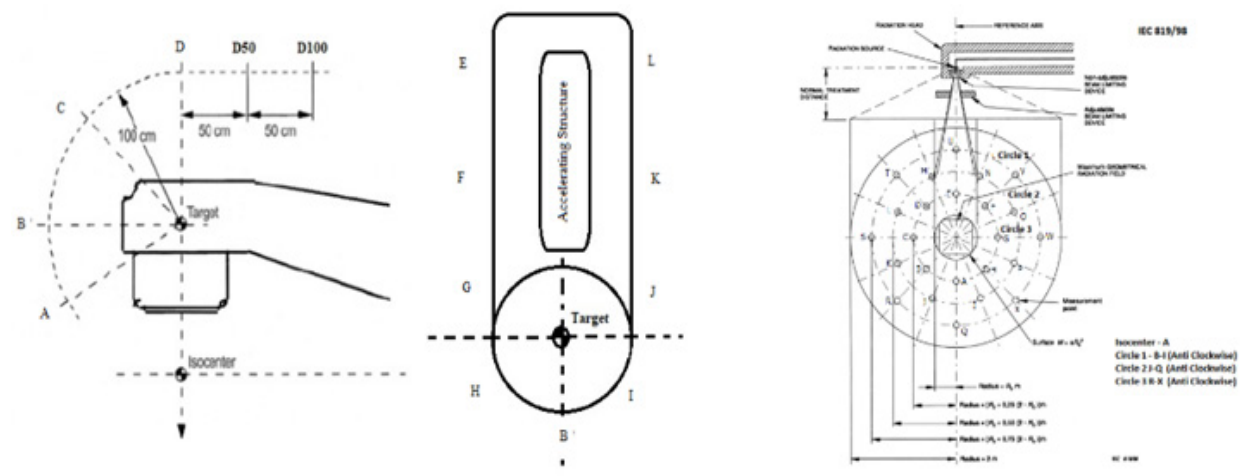

Figure 5. Schematic Diagram of Leakage Measurement Around the Treatment Head and Patient Plane 


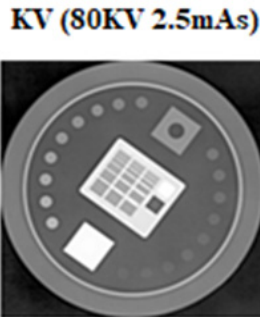

(a)

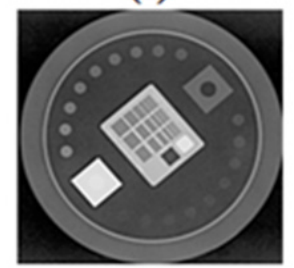

(d)
$2.5 \mathrm{MV}$

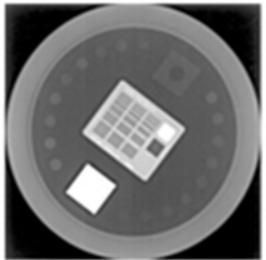

(b)

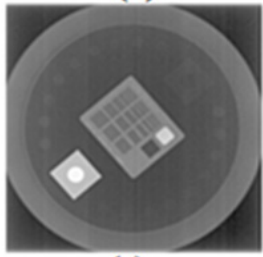

(e)
$6 \mathrm{MV}$

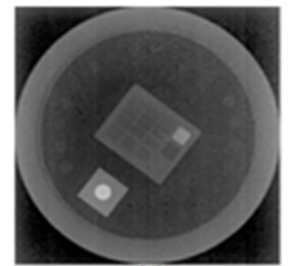

(c)

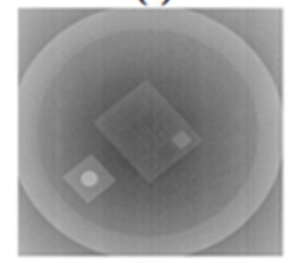

(f)

Figure 6. Spatial Resolution for without $(\mathrm{a}, \mathrm{b}, \mathrm{c})$ and with $(\mathrm{d}, \mathrm{e}, \mathrm{f})$ Scatter Medium for KV, 2.5MV and 6MV X-Ray Beams

increases above penumbra's lower definition of $20 \%$. An increase in percentage dose is observed of about $20 \%$ to $30 \%$ at $5 \mathrm{~cm}$ from field border for up to $20 \mathrm{~cm}$ depth. This increase in dose is due to an increase in photon beam softening as X-ray beam losses its energy at larger depths. These soft spectral x-ray photons are readily scattered at a larger angle which contributes more dose laterally beyond the filed border. Therefore, the patient out of field dose beyond the field border increases at larger depth for any field sizes for up to $30 \%$. It may cause an increase in normal structure dose that needs to be considered as a safety point of view depends on tolerance of the critical structures. Whereas, for $6 \mathrm{MV}$ flattened $\mathrm{x}$-ray beam, the out of filed dose increases from $2 \%$ to $6 \%$ and $5 \%$ to $13 \%$ for $5 \times 5 \mathrm{~cm}^{2}$ and $30 \times 30 \mathrm{~cm}^{2}$ field sizes at $5 \mathrm{~cm}$ from field border and from Dmax to until the depth of $20 \mathrm{~cm}$. Therefore, we need to analyse the critical organ dose at nearby field border in a real clinical situation at greater depths.

\section{Skin Dose}

Table 3 shows that the surface dose (basal layer $\sim 0.07$ $\mathrm{mm})$ measured $( \pm 0.1 \%)$ for $2.5 \mathrm{MV}$ imaging $\mathrm{x}$-ray beam with the help of parallel plate chamber (PPC40) as 88.15 $\%, 89.98 \%$ and $91.78 \%$ are high compared to $6 \mathrm{MV} \mathrm{FF}$ megavoltage x-ray beam surface doses $48.51 \%, 56.19 \%$ and $62.28 \%$ for the field sizes $10 \times 10 \mathrm{~cm}^{2}, 20 \times 20 \mathrm{~cm}^{2}$ and $30 \times 30 \mathrm{~cm}^{2}$ respectively. The skin dose measurement using RFA and CC13 chamber for $2.5 \mathrm{MV}$ x-ray beam shows that $87.82 \%, 90.0 \%$ and $91.32 \%$ for the same field sizes. A variation of $0.4 \%, 0.0 \%$ and $0.5 \%$ observed between CC13 and PPC40 chambers. A real skin dose which is dermal layer may be assessed at the depth $1.0 \mathrm{~mm}$ from table 3. At this depth, the dose increases to $95.35 \%, 95.86$ $\%$ and $96.5 \%$ for the field sizes $10 \times 10 \mathrm{~cm}^{2}, 20 \times 20 \mathrm{~cm}^{2}$ and $30 \times 30 \mathrm{~cm}^{2}$ respectively.

\section{Leakage Measurement}

Leakage measurements were done for leakage around the treatment head and in-patient plane. The head leakage measured $( \pm 0.001 \%)$ around the treatment head (Table 4) shows that the radiation level is well within the tolerance of $0.5 \%$ at $5 \mathrm{~cm}$ and $1 \mathrm{~m}$ from the treatment head respectively.

At the point $\mathrm{F}$ and $\mathrm{K}$, leakage radiation near the accelerating structure is comparable to that of points

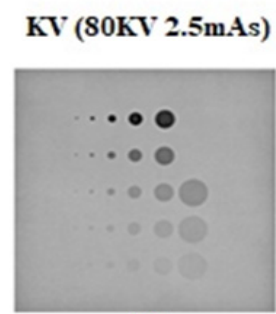

(a)

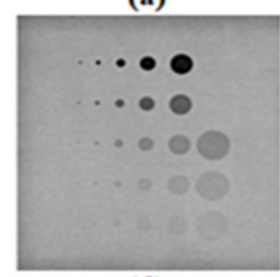

(d)
$2.5 \mathrm{MV}$

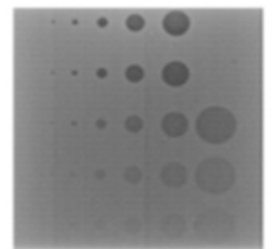

(b)

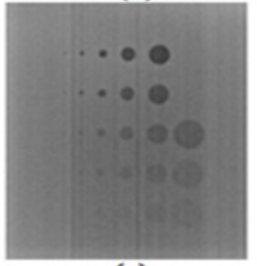

(e)
$6 \mathrm{MV}$

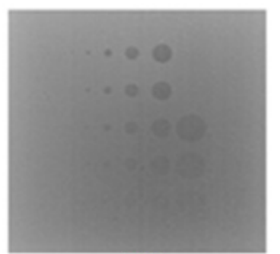

(c)

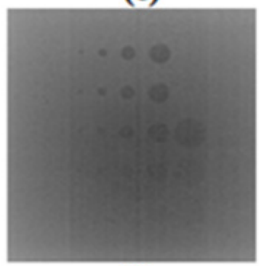

(f)

Figure 7. Contrast for without (a,b,c) and with (d,e,f) Scatter Medium of KV, 2.5MV and 6MV X-Ray Beams 
around the treatment head. At the points, G, H, I and $\mathrm{J}$, there is a slight increase in leakage radiation at $1 \mathrm{~m}$ compare to that of $5 \mathrm{~cm}$ due to more scattered radiation contributing from larger field of view.

Although the MUs required to take a $2.5 \mathrm{MV}$ image is relatively lower than that received during treatment, the absorbed dose at other than treatment area of body it needs to be analysed thoroughly as this low energy is prone to scatter readily and absorbed at skin level. The measured results tabulated in Table 5 shows that the leakage radiation in the patient plane is within the normal range of maximum dose and an average of $0.2 \pm 0.001$ $\%$ and $0.1 \pm 0.001 \%$ respectively at $0.71 \mathrm{~m}, 1.14 \mathrm{~m}$ and $1.57 \mathrm{~m}$ radius circle around the beam central axis at 100 $\mathrm{cm}$ isocenter level for $2.5 \mathrm{MV}$ and $6 \mathrm{MV}$ x-ray beams. However, the magnitude of leakage towards the gantry side at the points $\mathrm{E}, \mathrm{N}$, and $\mathrm{U}$ shows high in the patient plane for $2.5 \mathrm{MV}$ compare to $6 \mathrm{MV}$. In the case of imaging the pelvis or lower extremities, the patient's upper body will go towards the gantry side which will face higher leakage radiation in the head and shoulder region.

\section{MLC Transmission}

An incident of the patient imaging with the MLC aperture is large while taking the image with double exposure where the first image is normally taken with MLC aperture following a larger area to see the patient anatomy. Hence, it is vital to study the MLC transmission to know if the leakage radiation reaches the patient skin. The percentage of transmission for 2.5 MV megavoltage imaging $\mathrm{x}$-ray beam such as interleaf, intraleaf and edge effect are $0.40 \pm 0.01 \%, 0.37 \pm 0.01 \%$ and $11.0 \pm 0.01$ $\%$ compared to $6 \mathrm{MV}$ x-ray transmission of $1.5 \pm 0.01 \%$ $1.4 \pm 0.01 \%$ and $16.0 \pm 0.01 \%$ respectively.

\section{Contrast and Spatial Resolution}

Figure 6 shows that the spatial resolution of 2.0, 1.25 and $0.9 \mathrm{LP} / \mathrm{mm}$ and $2.0,1.0$ and $0.5 \mathrm{LP} / \mathrm{mm}$ without and with scatter condition for $\mathrm{KV}(80 \mathrm{KVp}, 2.0 \mathrm{mAs}), 2.5 \mathrm{MV}$ and $6 \mathrm{MV}$ x-ray beams respectively. Figure 7 shows that the contrast for $2.5 \mathrm{MV}$ x-ray beam is better than $6 \mathrm{MV}$ $\mathrm{x}$-ray beam. The mean energy of $2.5 \mathrm{MV}$ is less than that of the $6 \mathrm{MV}$ x-ray beam. However, the energy spectrum of $\mathrm{KV}$ is lower and the predominance of photoelectric effect is higher compared to $2.5 \mathrm{MV}$ x-rays.

\section{Discussion}

The mean energy calculated through mass attenuation coefficient from NIST XCOM data shows that it is in the range of kilovoltage energy level at the central axis and $15 \mathrm{~cm}$ off axis and there is a slight reduction of mean energy at $15 \mathrm{~cm}$ off axis. At these energies, predominance of photoelectric effect increases imaging contrast and spatial resolution. The absolute dose was calibrated to standardize and to know the dose absorbed during imaging procedure by knowing the number of MUs delivered during an imaging procedure. The output factor (Sc,p) shows that the $2.5 \mathrm{MV}$ imaging beam trends closely to Co-60. PDDs were studied and compared with other treatment energies. Unflatness of 2.5 MV x-ray beam was studied as per unflattened beam analysis protocol and symmetry was well below tolerance. The penumbra and out of field dose of 2.5 MV imaging x-ray beams are higher than other treatment beams which are used for imaging purpose. An increase in skin dose for 2.5 MV imaging beam was observed in comparison to the other imaging beam. Its losses concept of skin sparing effect; and it is significant when imaging is done on daily basis. Therefore, the skin dose needs to be analysed thoroughly and need to be considered in treatment dose.

The leakage radiation around the treatment head and at a patient plane is less than the normal range adopted by the competent authority. However, the leakage radiation in the patient plane towards the gantry side is slightly higher. It is a concern for patients imaged and treated in the pelvis and lower extremities during which patient's head is inevitably close to gantry and the resulting exposure to the head is higher than any other part of the body. The contrast and spatial resolution of the $2.5 \mathrm{MV}$ x-rays are much better than the $6 \mathrm{MV}$ x-ray beam used for imaging purpose but inferior to the kilovoltage $\mathrm{x}$-ray beam. The 2.5 MV x-ray imaging beam was analysed in view of beam characteristics and radiation safety to understand the above-studied concepts while using this imaging beam in a clinical situation. If the $2.5 \mathrm{MV}$-ray beam is used for treatment purpose in future, with increased dose rate, the above-studied concepts can be incorporated prior to implementation.

\section{Acknowledgments}

We thank Mr. Sunder from Kalyani Radiotherapy Specialty India (P) Ltd for helping us to design Perspex phantom and high $\mathrm{Z}$ materials for this project. We sincerely thank our Managing Trustee Dr. B. S. Srinath for his motivation in doing this work. This study id not funded by any organization.

\section{Statement Conflict of Interest}

There is no conflict of Interest

\section{References}

Abdullah R, Sapeei MS, Sukor NSA, et al (2018). Verification of relative output factor measurement using Gafchromic films for small-field radiosurgery photon beams. Mat Sci Eng, 298, 1-6.

Antonuk LE (2002). Electronic portal imaging devices: a review and historical perspective of contemporary technologies and research. Phys Med Biol, 47, 31-65.

Bel A, Van Herk M, Lebesque JV (1996) Lebesque. Target margins for random geometrical treatment uncertainties in conformal radiotherapy. Med Phys, 23, 1537-45.

Bentzen SM, Constine LS, Deasy JO, et al (2010). Quantitative Analysis of Normal Tissue Effect in the Clinic (QUANTEC): An introduction to the scientific issues. Int J Radiat Oncol Biol Phys, 76, 3-9.

Berger MJ, Hubbell JH (1987). XCOM: Photon cross-section database. NBSIR. (http://physics.nist.gov/xcom.)

Dawson L, Sharpe M (2006). Image-guided radiotherapy: rationale, benefits, and limitations. Lancet Oncol, 7, 848-58.

Devic S, Seuntjens J, Abdel-Rahman W, et al (2006). Accurate skin dose measurements using radiochromic film in clinical 
applications. Med Phys, 33, 1116-24.

Ding GX, Munro P (2018). The Characteristics of the newly available 2.5-MV imaging beam from a medical linear accelerator. Int J of Rad Oncol Biol Phys, 96, 698.

Eng TY (2000). Dose attenuation through a titanium alloy hip prosthesis. Med Dosim, 25, 7-8.

Gerward L, Guilbert N, Jensen KB, et al.(2004). WinX-Com: A program for calculating X-ray attenuation coefficients. Radiat Phys Chem, 71, 653-54.

Grace T, Christopher M, Daniel M, Edward S, Howard A (2016). Low-dose 2.5 MV cone-beam computed tomography with thick CsI flat-panel imager. J Appl Clin Med Phys, 17, 235-45.

Herman M, Ross A, Mayer R (1994). Clinical use of on-line portal imaging for daily patient treatment verification. Int $J$ Radiat Oncol Biol Phys, 28, 1017-23.

Jackson DF, Hawkes DJ (1981). X-ray attenuation coefficients of elements and mixtures. Phys Rep, 70, 169-233.

James LG, Jennifer O, Eduardo VBJ, et al (2016). Characterization of a $2.5 \mathrm{MV}$ inline portal imaging beam. J Appl Clin Med Phys, 17, 222-34.

Kerur BR, Thontadarya SR, Hanumaiah B (1993). Measurement of X-ray mass attenuation coefficient using mixed radiation emitters. Indian J Phys, 67, A-23.

Khan FM (1994). The Physics of Radiation Therapy. Williamson and Wilkins, Baltimore, 5, pp 62-70.

Marks J, Haus A, Sutton H, et al (1976). The value of frequent treatment verification films in reducing localization error in the irradiation of complex fields. Cancer, 37, 2755-61.

Mark R, Arnfield JV, Siebers Jong OK, et al (2000). A method for determining multi-leaf collimator transmission and scatter for dynamic intensity modulated radiotherapy. Med Phys, 27, 2231-41.

Michael GH, James MB, David AJ, et al (2001). Clinical use of electronic portal imaging: Report of AAPM radiation therapy committee task group 58. Med Phys, 28, 712-31.

Nithya K, Jabaseelan JS (2015). Assessment and evaluation of MV image guidance system performance in radiotherapy. Rep Pract Oncol Radiother, 20, 188-97.

Pisani L, Lockman D, Jaffray D, et al (2000). Setup error in radiotherapy: on-line correction using electronic kilovoltage and megavoltage radiographs. Int J Radiat Oncol Biol Phys, 47, 825-39.

Purdy JA, Biggs PJ, Bowers C, et al (1993). Medical accelerator safety considerations: report of AAPM Radiation Therapy Committee Task Group No: 35. Med Phys, 20, 1261-75.

Quanshi Z, Xiwen W, Qiyin S, et al (2016). Investigation and application of high megavoltage X-Ray imaging mode in radiotherapy. Int J Med Phys Clinic Engg Radiat Oncol, $\mathbf{5}, 42-50$.

Sahani G, Munish K, Dash Sharma PK, et al (2009). Compilation of Bhabhatron-II telecobalt unit with IEC standard-radiation safety. J Appl Clin Med Phys, 10, 120-30.

Sahani G, Sharma SD, Dash Sharma PK, et al (2014). Acceptance criteria for flattening filter-free photon beam from standard medical electron linear accelerator: AERB task group recommendations. J Med Phys, 39, 206-11.

Sauer OA, Wilbert J (2007). Measurement of output factors for small photon beams. Med Phy, 34, 1983-88.

Sharma SD, Reena S, Ujjwala M, et al (2013). Measurement of entrance skin dose for diagnostic x-ray radiographic examinations and establishment of local diagnostic reference levels. J Med Phy, 39, 860-63.

TRS No. 398 (2000). Absorbed Dose Determination in External Beam Radiotherapy: An International code of practice for Dosimetry on Standards of Absorbed Dose to Water. IAEA, Vienna, pp 61-72.
Verellen D, De Ridder M, Storme G (2008). A short history of image-guided radiotherapy. Radiother Oncol, 86, 4-13.

Weisner ME, Holden N, Coplen TB, et al (2013). Atomic weights of the elements 2011 (IUPAC technical report. Pure Appl Chem, 85, 1047-78.

Yin F, Wong J, Balter J, et al (2009). The Role of in-room kV $\mathrm{x}$-ray imaging for patient setup and target localization. American Association of Physicists in Medicine. Med Phys Pub Rep, 104, 20-30.

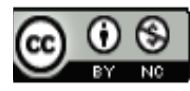

This work is licensed under a Creative Commons AttributionNon Commercial 4.0 International License. 